\title{
Diagnosing Sjögren's syndrome with salivary gland ultrasound
}

\begin{tabular}{|c|c|}
\hline $\begin{array}{l}\text { Dr Juan Carlos Nieto-González } \\
\text { from the Hospital General } \\
\text { Universitario Gregorio Marañón } \\
\text { has provided support for } \\
\text { the utilisation of salivary } \\
\text { gland ultrasound (SGUS) in } \\
\text { diagnosing and assessing } \\
\text { Sjögren's syndrome patients } \\
\text { in several published research } \\
\text { papers. When compared with } \\
\text { other diagnostic tools, SGUS is } \\
\text { non-invasive, widely available in } \\
\text { hospitals, and does not use any } \\
\text { ionising radiation. It can also } \\
\text { be used to diagnose juvenile } \\
\text { Sjögren's syndrome, which is a } \\
\text { rare and poorly defined disease. }\end{array}$ & $\begin{array}{l}\text { jögren's syndrome is a chronic } \\
\text { autooimmune disease, where } \\
\text { the immune system gets } \\
\text { overstimulated due to an unknown } \\
\text { origin and attacks healthy parts of the } \\
\text { body. In primary Sjögren's syndrome } \\
\text { (pSS), the immune system firstattacks } \\
\text { the glands that make tears and saliva, } \\
\text { the lacrimal and salivary glands } \\
\text { respectively, before manifesting in other } \\
\text { organs. Therefore, pSS is characterised } \\
\text { by lymphoytic infitration, which is } \\
\text { the build-up of white blood cells in } \\
\text { the body. The usual symptoms are dry } \\
\text { eyes and mouth with extra-glandular } \\
\text { manifestations, such as fatigue and pain } \\
\text { in the joints. In the UK, the syndrome }\end{array}$ \\
\hline
\end{tabular}

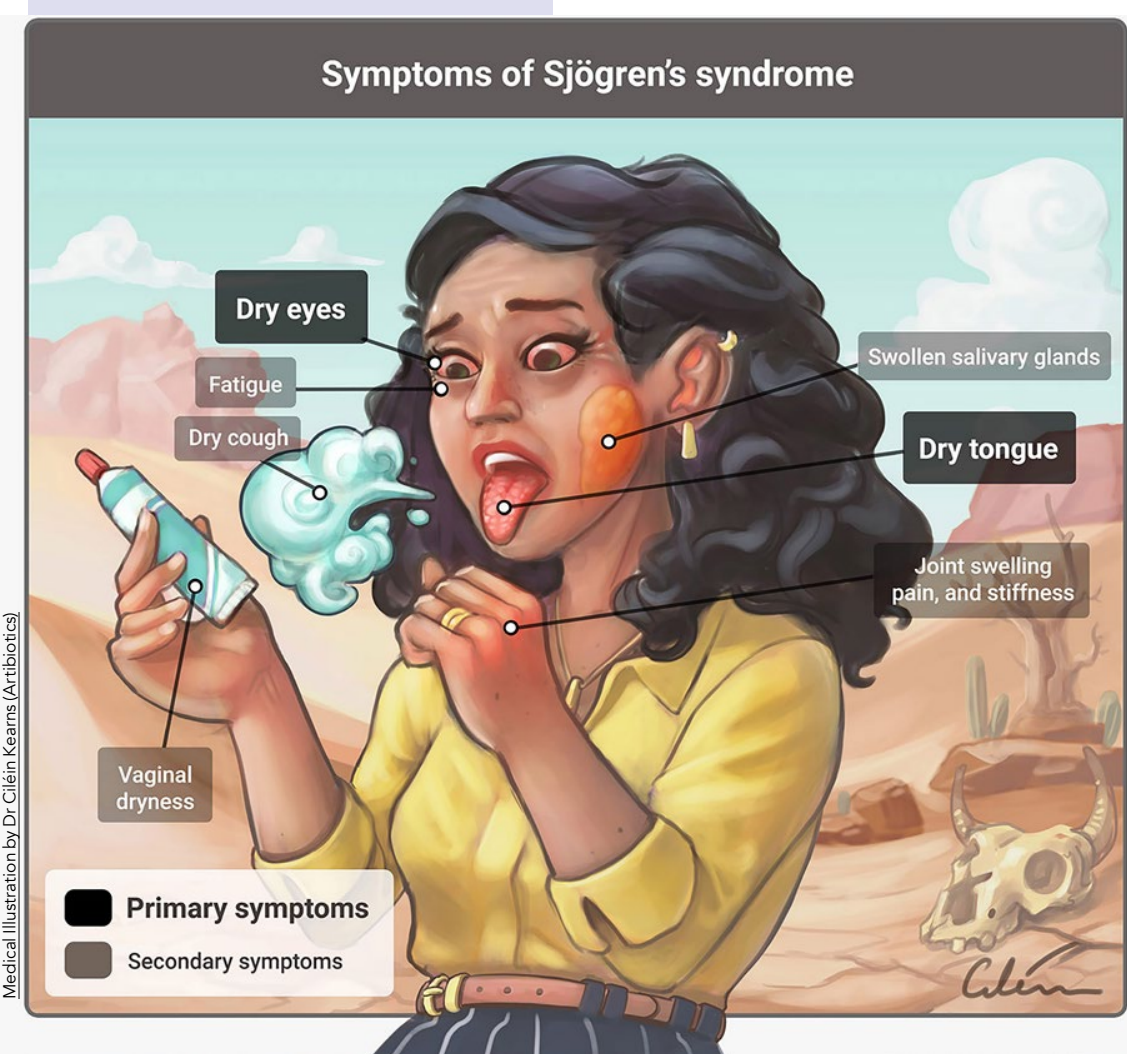

usually manifests in patients aged 40 60 years old. Globally, it is present general population

On the other hand, juvenile Sjögren's syndrome (jSS) is uncommon and adults. Patients are usually diagnosed at 10 years but challenges remain to ensure the diagnosis of patients is accurate. Recurrent inflammation of the parotid gland (parotitis) is the general sign at the onset of jSS. This symptom and the presence of characteristic autoantibodies, such as anti-Ro/La (anti-Sjögren's-syndromeelated antigen A autoantibodies) and RF (rheumatoid factor), are used as positive diagnostic tools in many patients. However, parotitis is common disorder in children and can be of bacterial or viral infectious origin instead. Therefore, patients who mistaknly dis as a symptom can be mistakeny diagnosed as contracting is later onset of some symptoms? which are dry eyes and mouth, in som younger patients and extra-glandular manifestations only occur in $50 \%$ of patients. Therefore, jSS is possibly an underdiagnosed disease, as both patients and doctors may not notice it until later in life.

Due to diverse clinical manifestations in younger patients, there is currently no available set criteria to diagnose jSS patients, and the American-European Consensus Group (AECG) or the American College of Rheumatology/ (ACR-EULAR) are neither sensitive nor specific for jSS diagnosis.

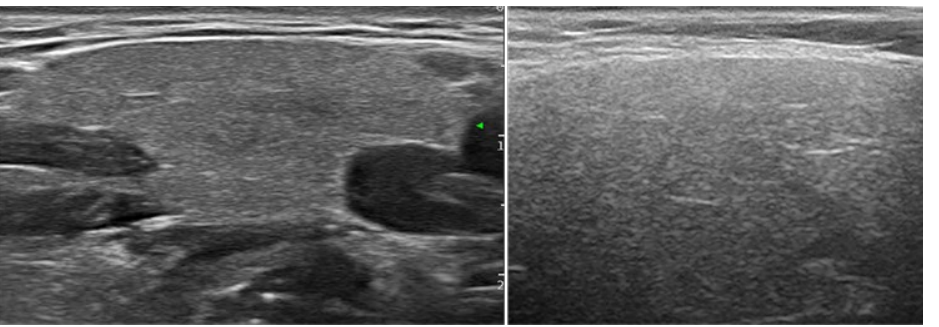

Longitudinal scan of normal
homogeneous parenchyma.

DIAGNOSIS OF

JÖGREN'S SYNDROME

At present, there is no available cure for

the disease. Many have hypothesised

the causes of the syndrome, from

genetics to hormone imbalance, bu

esearchers are unable to pinpoint a

specific cause. Treatments usually aim to manage the symptom
eye drops to keep eye drops to keep sas

such as sprays and

lozenges. Methods

of diagnosis

and assessment

Salivary gland ultrasoune, widely available, relatively

invasive, widely available, relatively cheap, and has a high resolution. The procedure can be repeated multiple and is available as an outpatient visit, which gives more flexibility for patients. Dr Juan Carlos Nieto-González is a s Sjögren's syndrome

For example, results from SGUS in multiple adult patients showed a consistent pattern, such as similar intermal echotexture (structure of include salivary flow measurement; minor salivary gland biopsy, sialography gland), scintigraphy (gan of the salivary and/or MR

In patients with the absence of characteristic autoantibodies, biops are usually performed, which is invasive, with low sensitivity and variable interobserver reliability. Patients with dry eyes and mouth (sicca symptoms) are assessed using salivary radioisotope attached to a drug and the subsequent gamma radiation emitted is measured.

In summary, these tools are invasive and involve ionising radiation, which remains a challenge for patients living with PSS or jSS. Therefore, a bottleneck remains to find a safe alternative to diagnose patients.

SALIVARY GLAND ULTRASOUND Salivary gland ultrasound (SGUS) evaluates the structure of the salivary glands by detecting changes in soun waves. It is an appealing alternative or can be used as a supplement to syndrome. This is because it is non-

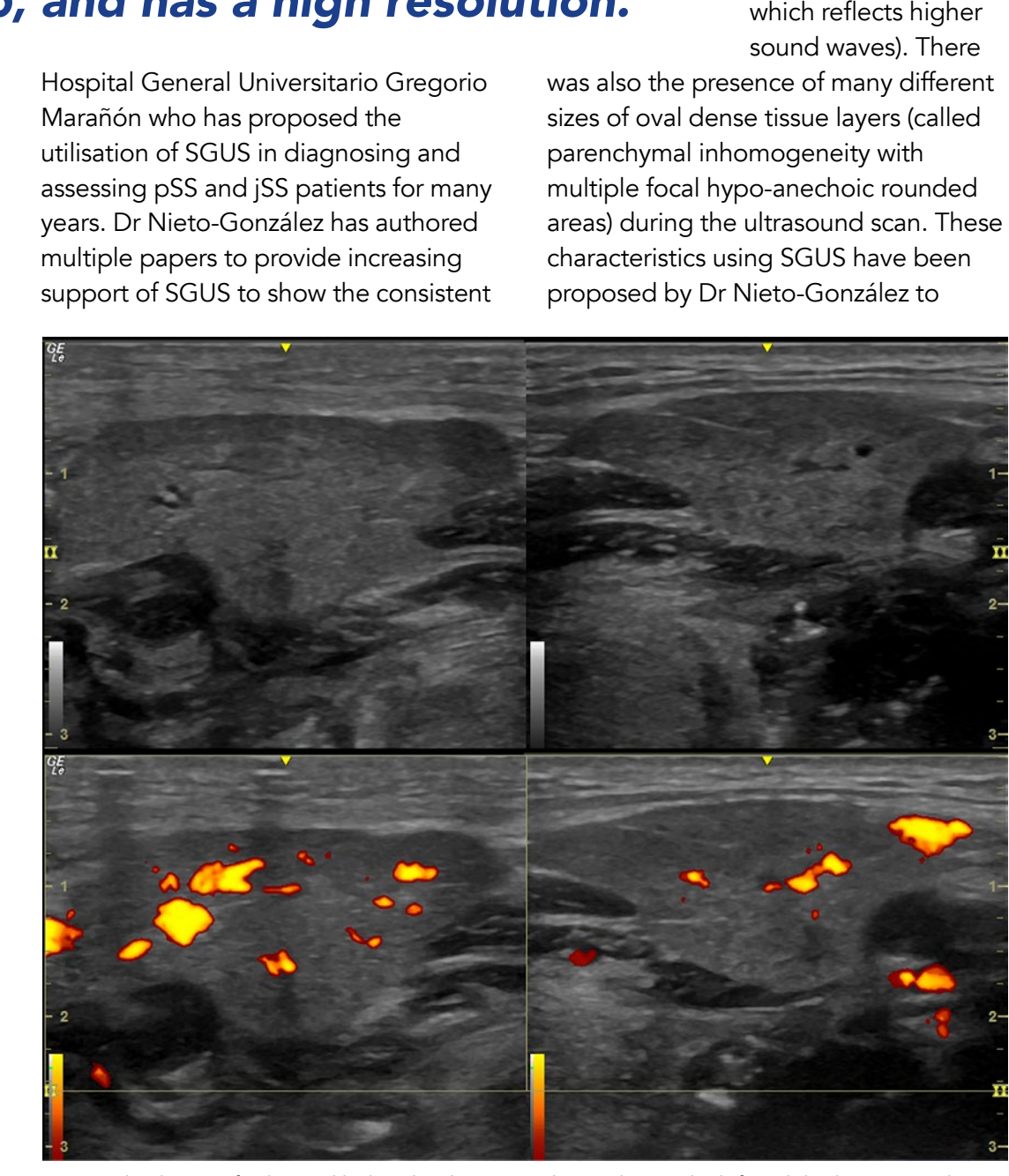
and echogenicity (signal intensity in ultrasounds; image at appears brighter wich reflects higher sond waves). There 


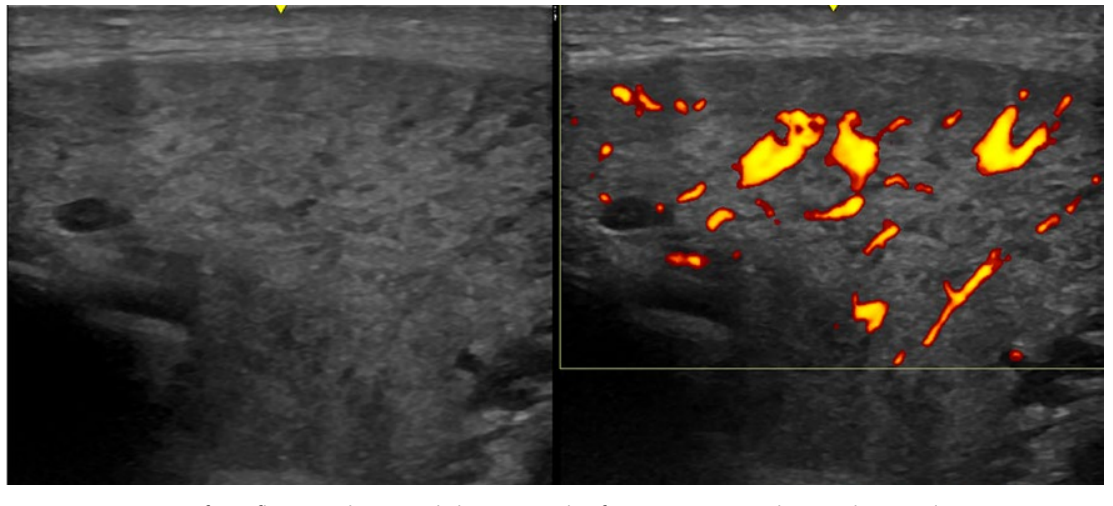

Tansverse scan of a inflammed parotid due to viral infection on B and Doppler mode.

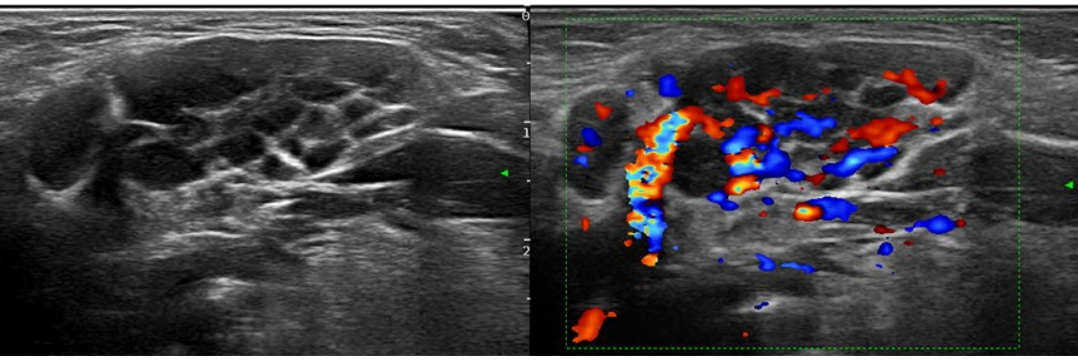

Tansverse scan of parotid gland on B and Doppler mode, with cyst and high Doppler signal.

be included in the diagnostic criteria

established pSS. This clinical study of $\mathrm{pSS}$, as they are consistent across observed in children with iSS, which may make the diagnostic criteria using SGUS applicable to all age groups. However, there are also other diseases that show similar pathologies, such as amyloidosis and lg4 syndrome.

Previous studies found that inclusion of SGUS information in the diagnosis

Salivary gland ultrasound may be a potential tool to reliably diagnose and assess primary and juvenile Sjögren's syndrome patients.

of $\mathrm{pSS}$ to the AECG criteria increased sensitivity from $77.9 \%$ to $87 \%$ while inclusion to the ACR criteria increased sensitivity from $64.4 \%$ to $84.4 \%$. These studies showed that SGUS may be a feasible and reliable diagnostic tool, which can be used alongside current diagnostic methods.

Dr Nieto-González and his colleagues aimed to assess multi-observer reliability of ultrasonographers with varying levels of working experin
using SGUS on patients with signals (measurement of blood velocity; increased blood flow and vascularisation are correlated with in PSS). This study showed that SGUS

\section{B Behind the Research}

\section{Dr Juan Carlos Nieto-González}

E: juancarlos.nietog@gmail.com T: +34637723549

is a reliable tool to assess the structure of salivary glands in pSS patients during reliability of SGUS in early diagnosis of pSS. More research is needed with larger patient groups, as these clinical studies have been conducted with a relatively small sample size.

As diagnosis criteria and symptoms for jSS have not been properly characterised in a large cohort of patients, Dr Nieto-González and other researchers conducted a clinical study, which was published in Arthritis Care \& Research in 2020. SGUS was performed on 67 patients with jSS from the onset of disease until they were 18 years old. The patients were of European and American nationalities, which ensures diversity he linical study to other regions of the world Approximately $63 \%$ f patients ( $n=42 / 67$ ) fulfilled either the AECG or ACR-EULAR criteria that are widely used to diagnose adult patients. In comparison, $61 \%$ of patients $(n=41 / 6)$ showed signs of pathology when using SGUS for diagnosis. Moreover, patients that showed Sjögren's syndrome pathology were positively associated with characteristic autoantibodies compared to patients who did not show Sjögren's-syndrome pathology. These findings showed that the presence of antibodies and SGUS can be used JSS patients. It can also be included in AECG and ACR-EULAR, narrowing the diagnosis of jSS.

In conclusion, these results showed that SGUS may be a potential tool to isS patients. However, the usage of ultrasound to diagnose and assess other connective tissues diseases have to be further investigated. The strong association between pathological SGUS and autoantibody positivity may determine a subpopulation of patients with potentially severe manage the disease. All in all, these the classification criteria of Sïgs in's syndrome in the futre. Sögren's together to increase the diagnosis of reliably diagnose and assess pSS and Sjögren's syndrome, which may help researchers better understand ways to results by DrNeto Gonzallez provide

\section{Research Objectives}

The research of Dr Juan Carlos Nieto-González centres on auto-immune diseases and chronic arthritis, both in adults and children.

\section{Detail}

Juan Carlos Nieto-González, Calle José Antonio Corrales 11, 4 D, 28055 Madrid, Spain

Bio

Dr Juan Carlos Nieto González is a consultant rheumatologist and fellowship tutor of the Universitario Gregorio Marañón, Madrid. He has a PhD in primary Sjögren's syndrome) and is Professor of the

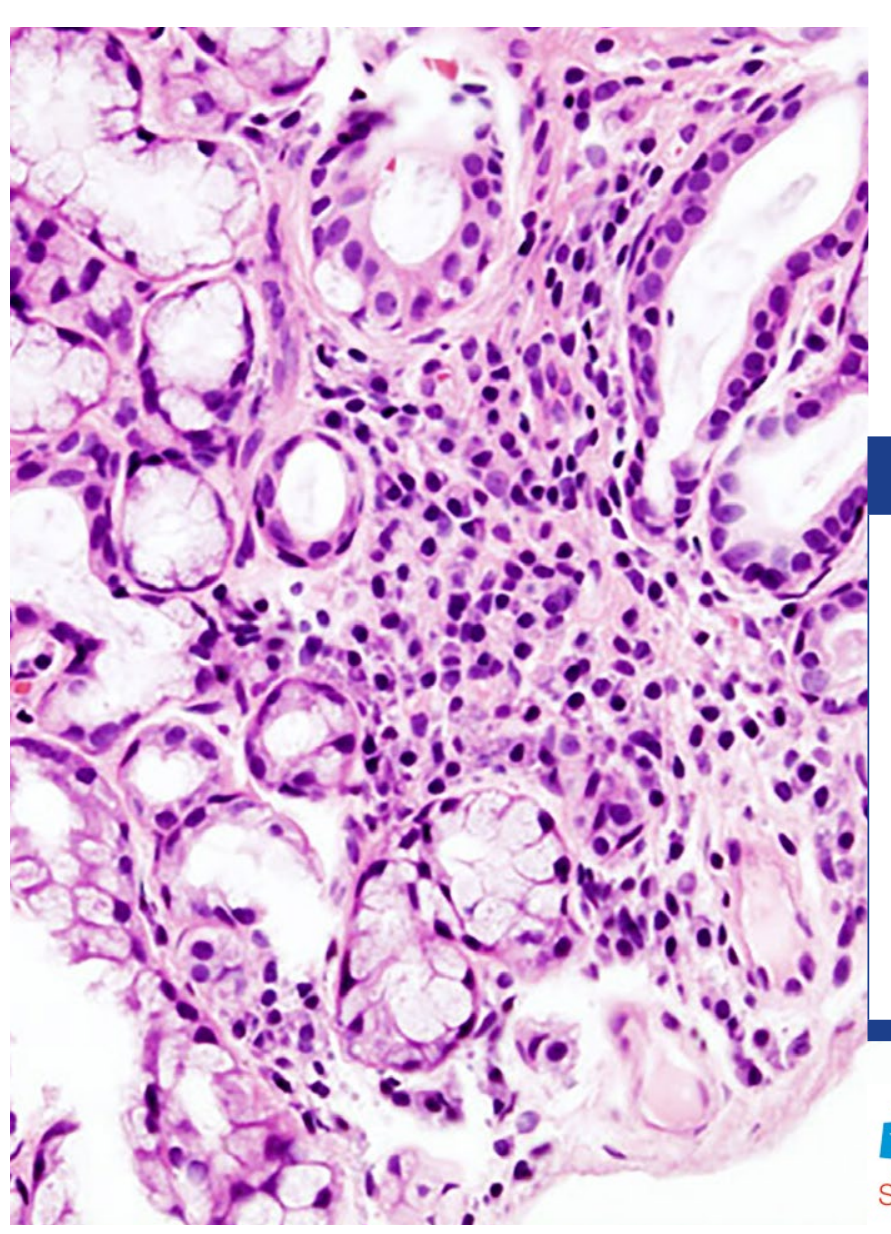
in medicine (his thesis was on salivary gland ultrasound

\section{References}

Damjanov, N., Milic, V., Nieto-González, J. C., Janta, I. et al. (2016). Multiobserver reliability of ultrasound assessment of salivary glands in patients with established primary Sjogren's syndrome. The Journal of Rheumatology. $43(10), 1858-1863$

Hammenfors, D. S., Valim, V., Bica, B. E. R. G., Pasoto, S. G. (2020). Juvenile Sjögren's syndrome: Clinical characteristics \& Research. $72(1), 78-87$

Jousse-Joulin, S., Nowak, E., Cornec, D, Brown, J. et al. (2017). Salivary gland ultrasound abnormalities in primary Sjögren's syndrome: Consensual US-SG core items definition and reliability. RMD Open. 3 (1).

Nieto-González, J. C., Monteaguado, I., Bello, N. Martínez-Estupiñan, L. et al. (2014). Salivary gland ultrasound in children: a useful tool in the diagnosis of

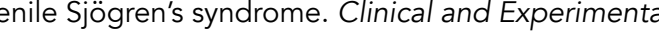
logy. 32, 578-580.

Nieto-González, J. C., Ovalles-Bonilla, J. G., Estrada, E., Serrano-Benavente, B. (2018). Salivary gland ultrasound is linked to the autoimmunity profle in patients with prim

\section{Personal Response}

What other areas of research are needed to further support the utilisation of SGUG in Sjögren's diagnosis?

To support the use of the SGUS in Sjögren's syndrome there are still many things to do. One of the most important is to confirm the graduation scale and There is also a need to evaluate the relation between manifestations and disease prognosis.
Hospital General Universitario Gregorio Marañón 